\title{
THE STRUCTURE OF SOME RIGHT NOETHERIAN RINGS WITH KRULL DIMENSION ONE
}

\author{
C. L. WANGNEO
}

(Communicated by Maurice Auslander)

\begin{abstract}
In this note we prove a structure theorem for a right Noetherian ring $R$ with krull dimension one having a right Artinian quotient ring and, moreover, that has a finitely generated, faithful, critical right module $U$ with krull dimension $U$ equal to one. We end this note with some examples that clarify certain features of this theorem.
\end{abstract}

\section{NOTATION AND TERMINOLOGY}

Let $R$ be a ring and $M$ a right $R$-module. We denote the right krull dimension of $M$ or $R$ by $|M|$ or $|R|$. For basic properties of krull dimension, the reader is referred to [6]. A module with krull dimension is said to be $\alpha$ krull homogeneous or simply krull homogeneous in the case where $|M|=\alpha$, if $|N|=|M|$, for every nonzero submodule $N$ of $M$. The ring $R$ is said to be krull homogeneous if the module $R_{R}$ is krull homogeneous. For the definition of Primary module or Primary ring and various results about Prime ideals associated to a module we refer the reader to Gordon [4]. If $A$ is a ring then its nilradical will be denoted by $N(A)$. The set of regular elements of $A$ is denoted by $C(O)$ and $C(B)$ denotes the set of elements of $A$ that are regular modulo $B$ where $B$ is an ideal of $A$. If $S_{i}, i \in I$, are subrings of a ring $R$ then their internal direct sum is denoted by $\dot{+}_{i \in I} S_{i}$. A ring $R$ is said to be nonsingular if the right singular ideal of $R$ is zero. The set of associated prime ideals of a right module $M$ is denoted by $\operatorname{Ass}(M)$. As usual rings are with identity and modules are unitary.

\section{MAIN THEOREM}

Proposition 1. Let $R$ be a right Noetherian ring with $M$ a faithful, finitely generated, critical module over $R$ such that $|M|=|R|$. Then $R$ is a krull homogeneous $P$-primary ring, where $P$ is the associated prime of $M$. Furthermore, if $I$ is an essential right ideal of $R$ then $|R / I|<|R|$.

Proof. For the proof of this proposition see Gordon [5, Lemma (1.7)] and Boyle and Feller [1, Corollary (2.5)].

Received by the editors September 6, 1990 and, in revised form, July 17, 1991.

1991 Mathematics Subject Classification. Primary 16-XX; Secondary 16P40, 16P50, 16 U20. 
Proposition 2. Let $R$ be a nonsingular, right Artinian ring that is $P$-primary for some prime ideal $P$ of $R$. Then $P=e R$ for some idempotent $e$ of $R$.

Proof. If $P=0$, then $e=0$ and so there is nothing to prove. So, suppose $P \neq 0$. Let $I$ be a right ideal maximal with the property that $I P=0 . I$ is clearly a two-sided ideal of $R$ and $P$ is a maximal ideal of $R$ because $R$ is right Artinian. Notice that by Gordon [4], $I$ is a large right ideal of $R$. So $I \nsubseteq P$ because $R$ is nonsingular. Also $P \nsubseteq I$ for otherwise $P=I$, and hence $P$ would be large. Hence $P+I=R$. Now one can easily verify that $P=e R$ for some nontrivial idempotent $e$ of $R$.

Theorem 3 (Main theorem). Let $R$ be a right Noetherian ring with $|R|=1$ and such that $R$ has a right Artinian quotient ring $Q$. If $U$ is a finitely generated, faithful, critical module over $R$ with $|U|=|R|$, and if Ass $U=P$ for some prime ideal $P$ of $R$, then the following hold.

(i) $P=e R$ for some idempotent $e$ of $R$.

(ii) $R=S+m \dot{+} T$, where $S$ is a right Artinian ring that is nonsingular and primary for some Prime ideal of $S, m$ is an ideal of $R$ with $m^{2}=0$, and $T$ is a nonzero prime ring such that $T \approx R / P$ with $|T|=1$.

(iii) $R=R_{1}+N(R)$ where $R_{1}$ is a Semiprime ring with $R_{1} \approx R / N(R)$.

Proof. If $P=0$ then there is nothing to prove. So we suppose that $P \neq 0$.

(i) By Proposition $1 R$ is krull homogeneous, $P$-Primary. Also $R$ is nonsingular. Let $I$ be a right ideal maximal with respect to the property $I P=0$. $I$ is clearly a two-sided ideal of $R$ that is also a large right ideal by Gordon [4]. Hence by Proposition $1 R / I$ is a right Artinian ring. Let $c \in C(0)$. Then $c r \in I \Rightarrow c r P=0$ and hence $r P=0$, which implies $r \in I$. So $c+I$ is a right regular element of $R / I$. Since $R / I$ is right Artinian, $c+I$ is a unit of $R / I$. Thus, if $Q_{i}, 1 \leq i \leq m$, are the Prime ideals of $R$ that are minimal over $I$, then $P \nsubseteq Q_{i}$. For if $P \subseteq Q_{i}$ then $Q_{i} / N$ is a nonminimal prime ideal of $R / N$ ( $N$ being the nilradical of $R$ ), and so $Q_{i} / N$ must contain a regular element of $R / N$. Since $R$ has a right Artinian quotient ring, so by Small's theorem [8] we get that $Q_{i}$ contains a regular element of $R$. By what we have done about this shows $Q_{i} / I$ is not a proper ideal of $R / I$. Hence we must have $P \nsubseteq Q_{i}$. Now we claim $I+P=R$. For if $I+P \neq R$, then there exists a maximal ideal $X$ of $R$ such that $I+P \subseteq X$. Since $R / I$ is a right Artinian ring, so $X / I=Q_{j} / I$ for some $j, 1 \leq j \leq m$. Hence $I+P \subseteq X$ implies $P \subseteq X$. But $X=Q_{j}$ shows $P \subseteq Q_{j}$, contradicting our above assertion that $P \nsubseteq Q_{j}$. Hence $I+P=R$. Combining this with $I P=0$, we get that there exists an $e \in R$, with $e^{2}=e$ and such that $e R=e P=P$.

(ii) See Chatters and Hajarnavis [3, Lemma 8.8, 6.13] for the proof of (ii)). More explicitly, $S=e R e, m=e R(1-e)$, and $T=(1-e) R(1-e)$. If $I$ is as in (i) above then $I=R(1-e)$ and $S \approx R / I$, which implies that $S$ is a right Artinian ring.

(iii) $R$ is nonsingular implies that $S$ is nonsingular. Also $U S$ is a finitely generated, faithful module over $S$ that is also a uniform $S$ module because $S$ is nonsingular. Thus $S$ is $X$-Primary for some prime ideal $X$ of $S$. Since $S$ is a right Artinian ring, by Proposition $2 X$ is generated as a right ideal of $S$ by an idempotent of $S$. It is easy to show by a Noetherian Induction and Chinese remainder theorem as in (i) that $S=S_{1}+N(S)$ where $S_{1}$ is a semiprime ring 
and where $N(S)=$ nilradical of $S$. Also $S / N(S) \approx S_{1}$. Now by (ii) either

$$
R=S \dot{+} m \dot{+} T \text { or } R=S_{1} \dot{+} N(S) \dot{+} m \dot{+} T
$$

or

$$
R=\left(S_{1} \dot{+} T\right) \dot{+}(N(S) \dot{+} m) .
$$

If $N=$ nilradical of $R$, then it is easy to see that $R / N(R) \approx S_{1}+T$. So if $R_{1}=S_{1} \dot{+} T$ then $R_{1}$ is a semiprime ring and $N(S) \dot{+} m=N$. Thus $R=R_{1} \dot{+} N$ where $R_{1} \approx R / N$.

Remark. Because of Theorem 3(i), the ring $R$ can be put isomorphic to a ring $A$ where $A=\left[\begin{array}{ll}S & m \\ 0 & T\end{array}\right]$, where $S$ is a right Artinian subring of $R, m$ is an ideal of $R$ with $m^{2}=0$, and $T \approx R / P$ is a nonzero prime factor ring of $R$.

We now end this note with some examples. Throughout here we assume $F$ is a field and $F[x]$ the usual polynomial ring.

Example 1. There exists a ring $R$ satisfying the conditions of the main theorem and yet $R$ is not a prime ring.

Consider

$$
R=\left[\begin{array}{ll}
F & F[x] \\
0 & F[x]
\end{array}\right]
$$

Notice that $R$ has a finitely generated, faithful, critical, right module

$$
M=\left[\begin{array}{cc}
F & F[x] \\
0 & 0
\end{array}\right]
$$

with $|M|=|R|=1$. Clearly $R$ is not a prime ring and $R$ is right Noetherian but not left Noetherian.

Example 2. There exists a ring $R_{1}$ that is right Noetherian of krull dimension one and, moreover, that has a finitely generated, faithful, critical module $M$ with $|M|=\left|R_{1}\right|$ and yet $R_{1}$ does not have an Artinian quotient ring. Let

$$
R_{1}=\left\{\left[\begin{array}{cc}
a & g(x) \\
0 & a_{+}(x)
\end{array}\right] \mid \begin{array}{c}
g(x) \in F[x], \quad a \in F, \\
(x)=c x \mid c \in F[x]
\end{array}\right\} \subseteq R
$$

where $R$ is as in Example 1. Notice that $\left|R_{1}\right|=1$. Also

$$
M=\left[\begin{array}{cc}
F & F[x] \\
0 & 0
\end{array}\right]
$$

is a finitely generated, faithful, critical module over $R_{1}$ with $|M|=1$. Notice that

$$
P=\left\{\left[\begin{array}{cc}
0 & g(x) \\
0 & 0
\end{array}\right] \mid g(x) \in F[x]\right\}
$$

is a prime ideal of $R_{1}$ that is associated to $R_{1}$ as well as to $M$. Let

$$
Q=\left\{\left[\begin{array}{cc}
0 & g(x) \\
0 & (x)
\end{array}\right] \mid \begin{array}{c}
g(x) \in F[x] \\
(x)=c x \mid c \in F[x]
\end{array}\right\} .
$$

Then $Q$ is a maximal ideal of $R_{1}$ with $P \nsubseteq Q$. Also $Q P=0$. clearly $R_{1}$ cannot have an Artinian quotient ring. 
Example 3. One may be tempted to think that $S$ is a prime ring in the main theorem (Theorem 3). We give an example to show that $S$ is not a prime ring. Observe first that if $S$ is a prime ring then there would exist in $R$ a minimal prime ideal $Q(\neq P)$ such that $Q P=0$ showing, thereby, that $\min \operatorname{Spec}(R)=$ $\{Q, P\}$, where by $\min \operatorname{Spec}(R)$ we mean the set of minimal prime ideals of $R$.

However, we will give an example of a ring $A$ satisfying all the conditions of Theorem 3 whose $\min \operatorname{Spec}(A)$ contains more than two prime ideals. Let

$$
R=\left[\begin{array}{cc}
F & F[x] \\
0 & F[x]
\end{array}\right], \quad P=\left[\begin{array}{cc}
F & F[x] \\
0 & 0
\end{array}\right], \quad Q=\left[\begin{array}{ll}
0 & F[x] \\
0 & F[x]
\end{array}\right] .
$$

Clearly $P$ is a critical right ideal of $R$ that is finitely generated and faithful over $R$ with $|P|=|R|=1$. Also $\min \operatorname{Spec}(R)=\{P, Q\}$. Notice that $R$ has a right Artinian quotient ring. Consider $A=\left[\begin{array}{ll}F & P \\ 0 & R\end{array}\right]$. Then $A$ satisfies all the conditions of Theorem 3 with $U=\left[\begin{array}{ll}F & P \\ 0 & 0\end{array}\right]$ is a finitely generated, faithful, critical module over $A$ with $|U|=|A|=1$. Notice that

$$
\min \text { Spec }=\left\{\left[\begin{array}{ll}
F & P \\
0 & P
\end{array}\right],\left[\begin{array}{ll}
F & P \\
0 & Q
\end{array}\right],\left[\begin{array}{ll}
0 & P \\
0 & R
\end{array}\right]\right\} .
$$

Hence there are more than two elements in $\min \operatorname{Spec} A$.

Remarks. We remark that it is an open question whether or not $A$ is a Prime ring if $A$ is supposed to be a Noetherian ring and, moreover, that has a finitely generated, faithful, critical right module $M$. This question is equivalent to the question of whether or not the nilradical of $A$ is weakly ideal invariant. For this question and related results see [2,7]. We mention that the above question is open even when we assume that $A$ has an Artinian quotient ring. In view of this it becomes desirable to prove our main theorem, namely, Theorem 3 for the ring $R$ of this theorem, when $R$ is assumed to have krull dimension greater than one. However, we are unable to prove this result.

\section{REFERENCES}

1. A. K. Boyle and E. H. Feller, Semi critical modules and K-Primitive rings, lectures in Module theory, Lecture Notes in Math., vol. 700, Springer-Verlag, New York, 1979, pp. 57-74.

2. K. A. Brown, T. H. Lenagan, and J. T. Stafford, Weak ideal invariance and localisation, J. London Math. Soc. (2) 21 (1980).

3. A. W. Chatters and C. R. Hajarnavis, Rings with chain conditions, Pitman, London, 1980.

4. R. Gordon, Primary decomposition in right noetherian rings, Comm. Algebra (1974), 491524.

5. Some aspects of non-commutative noetherian rings, Lecture Notes in Math., vol. 545. Springer-Verlag, New York, 1975, pp. 105-127.

6. R. Gordon and J. C. Robson, Krull dimension, Mem. Amer. Math. Soc., vol. 133, Amer. Math. Soc., Providence, RI, 1973.

7. G. Krause, T. H. Lenagan, and J. T. Stafford, Ideal invariance and Artinian quotient rings, J. Algebra 55 (1978), 145-154.

8. L. W Small, Correction and addendum 'Orders in Artinian rings', J. Algebra 4 (1966), 505-507.

Department of Mathematics, University of Jammu, New Campus, Jammu-180004, J \& K STATE, INDIA 